\title{
Computational analysis of an aortic valve jet with Lagrangian coherent structures ${ }^{1}$
}

\author{
Shawn C. Shadden ${ }^{1}$, Matteo Astorino ${ }^{2} \&$ Jean-Frédéric Gerbeau ${ }^{2}$ \\ ${ }^{1}$ Mechanical and Aerospace Engineering, Illinois Institute of Technology, Chicago, IL \\ ${ }^{2}$ INRIA Rocquencourt, Le Chesnay Cedex, France
}

\begin{abstract}
Important progress has been achieved in recent years in simulating the fluid-structure interaction around cardiac valves. An important step in making these computational tools useful to clinical practice is the development of postprocessing techniques to extract clinically-relevant information from these simulations. This work focuses on flow through the aortic valve and illustrates how the computation of Lagrangian coherent structures can be used to improve insight into the transport mechanics of the flow downstream of the valve, towards the goal of aiding clinical decision making and the understanding of pathophysiology.
\end{abstract}

Key Words: Aortic valve, Finite-time Lyapunov exponents, Fluid-structure interaction, Hemodynamics, Lagrangian coherent structures.

${ }^{1}$ published in Chaos, Vol. 20, p. 017512, 2010 
The computation of Lagrangian coherent structures, LCS, has become an important method for examining unsteady fluid transport. This method entails computing special moving boundaries (LCS) in the fluid domain, which can be used to reveal dominant flow features, such as vortex boundaries or separation profiles, or uncover kinematic processes organizing fluid mixing. Knowledge of transport mechanics in the cardiovascular system is particularly compelling. Disturbed flow conditions, including vortical or separated flow, are known to influence health maintenance and disease progression. Therefore, strong motivation exists to utilize LCS to better understand transport in the cardiovascular system. Herein, we tackle the problem of characterizing flow through the aortic valve. Each cardiac cycle a jet of oxygenated blood is ejected from the heart to the aorta. The diagnoses of a common valvular heart disease, aortic stenosis, is based on measuring the size of the aortic valve jet. In this paper we employ a coupled fluid-structure interaction scheme to simulate flow through a realistic deformable, $3 \mathrm{D}$ aortic valve model and use this data to compute LCS. This approach enables a more precise measure of the jet size than existing methods and an improved understanding of the jet geometry and dynamics.

\section{Introduction}

The aortic valve releases pressurized blood from the left ventricle into the ascending aorta during the systolic phase of the cardiac cycle and prevents possible back flow during the diastolic refilling phase. In normal anatomy, this valve is composed of three semilunar leaflets (cusps) that passively move apart or mate together in response to the pressure gradients imposed by the blood pumped from the heart.

Among the pathologies that may affect the functioning of the valve, aortic stenosis (AS) is one of the most common. AS is an abnormal narrowing of the aortic valve opening, which can result from various causes such as calcification, congenital or rheumatic diseases [18, 4]. Depending on the severity of the AS, different medical treatments are used. For example, valve replacements are usually recommended for severe stenoses, and for mild or moderate stenoses, therapies to control symptoms and restriction of strenuous activities are often advised.

Over the past years, the medical community broadly accepted that reliable assessment of AS requires estimates of the aortic valve area (AVA) [18]. Direct visualization of the anatomical 
area of the stenotic orifice, the so-called geometric orifice area (GOA), has been considered a theoretically ideal way to assess the AS. Nonetheless, as observed in [3], proper delineation of the orifice circumference by means of image-based planimetry has been found to be difficult and moreover it has been noticed that GOA doesn't characterize the flow properties related to the stenosis. Hence, the development of good clinical indices to accurately assess the AS is still an active research field.

Depending on the diagnostic technique employed, different indices to calculate the AVA have been developed-the most popular among them being based on the Gorlin formula [21] and the continuity equation [32]. The Gorlin formula requires an evaluation of the pressure gradient across the valve to estimate the AVA. The index, called Gorlin area, can be obtained either with invasive measurements of pressure, done with micromanometer catheters, or by applying the classical Bernoulli equation to Doppler velocity measurements. Instead the continuity equation, based on the law of conservation of mass, provides an estimate of the AVA, the so-called effective orifice area (EOA), from noninvasive Doppler echocardiography measurements of the blood velocity.

Although the GOA, the Gorlin area and the EOA were initially believed to provide a similar estimation of the AVA, extensive comparison between the three quantities (e.g. see $[15,8]$ ) revealed important differences among them. As a matter of fact, the Gorlin formula and the continuity equation asses the stenosis severity taking into account the associated flow properties with some basic physical principles, such as Torricelli's law, Bernoulli's law and conservation of mass, while the GOA is a purely geometrical measure. In practice, the Gorlin area and the EOA provide an estimate of the minimal cross-sectional area of the jet formed downstream of the valve by the blood ejected during systole (Fig. 1). We refer to [19] for a detailed discussion about these indices.

The importance of the flow dependance in AS characterization has been widely investigated and it is now well-established. As a consequence, the Gorlin area and the EOA are usually preferred to the GOA, which is no longer considered a reliable index for AS. Important efforts are currently underway on improving clinical indices to better diagnose AS. Among them, for example, is the energy loss coefficient, $\mathrm{E}_{\mathrm{L}} \mathrm{Co}$, proposed in [20], which reflects the energy loss induced by the aortic stenosis and aims to better describe the increased overload imposed on the left ventricle.

In this work, we focus on the EOA, which is the most common index for the assessment of AS, as it can be derived from noninvasive Doppler echocardiography [3]. This index aims to asses AS by quantifying the size of the jet of ejected fluid from the heart. However, as a downside, it does not provide any direct, physical understanding of the actual geometry and dynamics of the jet. 
The analysis in this paper demonstrates a computational method to directly measure the jet size, shape and dynamics, which we believe provides a better and more clinically-relevant description of the jet.

The application of mathematical models and numerical tools to assess AS is a rather new field. In previous work, reduced models and simplified multi-dimensional models $[13,17]$ have been proposed to test the validity and quality of clinical indices. More recently, progress in numerical simulation of fluid-structure interaction (FSI) around cardiac valves (see for example $[27,12,41,44,14,1]$ ) has enabled multi-dimensional FSI models to be applied to the analysis of aortic valve stenosis $[22,7,39]$. In [39], instantaneous Eulerian measures obtained from a 3D FSI model of the aortic valve were used to compare different clinical indices for various stenotic geometries. Instantaneous Eulerian metrics are convenient since they can be directly obtained from the FSI simulations, but many important flow features are more easily comprehended using Lagrangian metrics.

In this work we utilize the computation of LCS to post-process results from multi-dimensional FSI simulations of the aortic valve. This framework enables a clearer understanding of the transient transport structures and mechanics of the flow than visualization of instantaneous Eulerian quantities. Previous work has shown the utility of LCS computations in complex cardiovascular flows $[36,46]$. In the particular case of aortic valve stenosis, LCS can characterize flow separation downstream of the valve and identify the time-dependent bounding surface of the blood flow jet. As a consequence, a precise measure of the "effective orifice area", or jet size, can be evaluated.

The paper is organized as follows: In $\S 2.1$, the mathematical models for the fluid and structure (valve and vessels), and the numerical challenges of the FSI coupling scheme will be briefly addressed. Next, a detailed description of computation of the Finite-Time Lyapunov Exponent field and LCS will be provided in $\S 2.2$. In $\S 3$, two different test cases-one $2 \mathrm{D}$ and one $3 \mathrm{D}$-will be used to illustrate the computational framework. A discussion on the results is contained in $\S 4$.

\section{Methods}

\subsection{Fluid-structure interaction in the aortic valve}

The modeling and simulation of the interaction between the blood and the valve is challenging due to the complex valve dynamics, possible contact amongst the valve leaflets, intrinsic flow unsteadiness and intense velocity and pressure gradients. Here we briefly describe the mathematical models and the numerical methods used to address these challenges. 


\subsubsection{Fluid and structure models}

In this study, we consider the fluid-structure model sketched in Fig. 2, where the flexible valve leaflets are surrounded by an homogeneous, viscous and incompressible fluid in a fixed domain $\Omega^{\mathrm{f}} \subset \mathbb{R}^{d}, d=2,3$. The assumption of a rigid aortic wall is made to limit the computational costs and complexity and is assumed reasonable considering the large stiffness of the wall compared to the leaflets.

Blood is a complex fluid, sometimes exhibiting non-Newtonian behaviors such as shear thinning, thixotropy and viscoelasticity [37]. In large arteries, however, it is widely accepted to consider a Newtonian-like behavior [47]. Herein blood is modeled as a Newtonian fluid governed by the incompressible Navier-Stokes equations

$$
\begin{array}{r}
\rho^{\mathrm{f}}\left(\frac{\partial \boldsymbol{u}}{\partial t}+\boldsymbol{u} \cdot \nabla \boldsymbol{u}\right)-\operatorname{div} \boldsymbol{\sigma}^{\mathrm{f}}(\boldsymbol{u}, p)=\mathbf{0}, \text { in } \Omega^{\mathrm{f}}, \\
\operatorname{div} \boldsymbol{u}=0, \text { in } \Omega^{\mathrm{f},}
\end{array}
$$

where $\boldsymbol{u}$ represents the velocity of the fluid, $p$ the pressure and $\boldsymbol{\sigma}^{\mathrm{f}}(\boldsymbol{u}, p) \stackrel{\text { def }}{=} 2 \mu \boldsymbol{\epsilon}(\boldsymbol{u})-p \mathbf{I}$ the Cauchy stress tensor, with $\boldsymbol{\epsilon}(\boldsymbol{u}) \stackrel{\text { def }}{=} \frac{1}{2}\left(\nabla \boldsymbol{u}+\nabla^{T} \boldsymbol{u}\right)$ and I denoting the strain tensor and identity tensor, respectively. The fluid density $\rho^{\mathrm{f}}$ and the viscosity $\mu$ are assumed constant and equal to $1.0 \mathrm{~g} \mathrm{~cm}^{-3}$ and $0.035 \mathrm{~g} \mathrm{~cm}^{-1} \mathrm{~s}^{-1}$, respectively. In solving (1), we impose the following conditions on the boundary, $\partial \Omega^{\mathrm{f}} \stackrel{\text { def }}{=} \Gamma_{\text {in }} \cup \Gamma_{\text {wall }} \cup \Gamma_{\text {out }}$, of fluid domain $\Omega^{\mathrm{f}}$ :

$$
\begin{aligned}
\boldsymbol{u} & =\mathbf{g}, \quad \text { on } \Gamma_{\text {in }} \subset \partial \Omega^{\mathrm{f}}, \\
\boldsymbol{u} & =\mathbf{0}, \quad \text { on } \quad \Gamma_{\text {wall }} \subset \partial \Omega^{\mathrm{f}}, \\
\boldsymbol{\sigma}^{\mathrm{f}}(\boldsymbol{u}, p) \cdot \mathbf{n}^{\mathrm{f}} & =R \int_{\Gamma_{\text {out }}} \boldsymbol{u} \cdot \mathbf{n}^{\mathrm{f}} d \gamma, \quad \text { on } \quad \Gamma_{\text {out }} \subset \partial \Omega^{\mathrm{f}} .
\end{aligned}
$$

At the inlet, $\Gamma_{i n}$, the vector function $\mathbf{g}$ maps the volumetric flow to a time-varying parabolic profile. On the arterial wall, $\Gamma_{\text {wall }}$, a no-slip boundary condition is imposed. Equation (4) defines a resistance-like boundary condition at the outlet, $\Gamma_{\text {out }} ; \mathbf{n}^{\mathrm{f}}$ is the outward normal on $\partial \Omega^{\mathrm{f}}$ and $R$ is the resistance, which represents the physical resistance imposed by the downstream arterial bed (for more details, see for example [42]).

The leaflets, in view of their thickness/size ratio, are modeled as co-dimensional one structures. For 2D FSI simulations, an inextensible 1D solid with deformation energy

$$
\mathcal{W}=\frac{1}{2} \int_{0}^{L} E I\left|\frac{\partial^{2} \boldsymbol{x}}{\partial s^{2}}\right|^{2} d s
$$


has been used for the leaflets. In Eq. (5), $\boldsymbol{x}(s)$ represents the position vector of a point along the structure, and the quantities $L, E$ and $I$ denote, respectively, the length, Young's modulus and the moment of inertia of the leaflet. We refer to [14] for more details on the model.

For 3D FSI simulations, the MITC4 general shell element model has been used for the leaflets $[9,1]$. The mechanics of the valve is described by a generalized Hook law with internal energy given by

$$
\mathcal{W}=\frac{1}{2} \int_{\hat{\Omega}^{\mathrm{s}}}\left[\mathcal{C}^{\alpha \beta \lambda \mu} e_{\alpha \beta} e_{\lambda \mu}+\mathcal{D}^{\alpha \lambda} e_{\alpha z} e_{\lambda z}\right] d V
$$

In Eq. (6), $\hat{\Omega}^{\mathrm{s}}$ represents the reference configuration for the solid, while $\boldsymbol{e}=\left(e_{\alpha \beta}\right)$ denotes the nonlinear Green-Lagrange strain tensor. The Greek symbols vary from 1 to 2 and are used for the tangential components to the surface, while $z$ denotes the normal direction. Lastly,

$$
\begin{gathered}
\mathcal{C}^{\alpha \beta \lambda \mu}=\frac{E}{2(1+\nu)}\left(g^{\alpha \lambda} g^{\beta \mu}+g^{\alpha \mu} g^{\beta \lambda}+\frac{2 \nu}{1-\nu} g^{\alpha \beta} g^{\lambda \mu}\right) \\
\mathcal{D}^{\alpha \lambda}=\frac{8 E}{t_{s}^{2}(1+\nu)} g^{\alpha \lambda}
\end{gathered}
$$

where $\nu$ is the Poisson ratio, $t_{s}$ the thickness and $g^{\alpha \lambda}$ the contravariant components of the metric tensor. We refer to [9] for more details on this shell model.

Remark 1 More complex structure models exist, which take into account peculiar characteristics of the valve such as the fibrous tissue network (see e.g. [43]). Nevertheless, in light of the scope our investigation, the model used herein is considered sufficient to illustrate the computational techniques under analysis.

\subsubsection{The coupled FSI problem: Formulation and numerical discretization}

For the current setup, namely a coupled FSI problem with an immersed solid having one dimension less than the fluid, the structure domain $\Omega^{\mathrm{s}}(t)$ coincides with the fluid-structure interface $\Sigma(t)$. Therefore, the coupling between the two problems is enforced through the following conditions

$$
\boldsymbol{u}=\partial_{t} \boldsymbol{d} \quad \text { and } \quad \llbracket \boldsymbol{\sigma}^{\mathrm{f}} \rrbracket \cdot \mathbf{n}^{\mathrm{s}}=\boldsymbol{\sigma}^{\mathrm{s}} \cdot \mathbf{n}^{\mathrm{s}} \quad \text { on } \quad \Sigma(t)
$$

Here, $\boldsymbol{d}$ and $\boldsymbol{\sigma}^{\mathrm{s}}$ represent, respectively, the displacement and the Cauchy stress tensor for the structure, while $\llbracket \boldsymbol{\sigma}^{\mathrm{f}} \rrbracket \cdot \mathbf{n}^{\mathrm{s}}$ is the jump of the hydrodynamic stress through the valve, with $\mathbf{n}^{\mathrm{s}}$ the normal on $\Sigma(t)$.

Different mathematical formulations for the coupled fluid-structure problem can be roughly divided in two families: moving domain and fixed domain methods. In moving domain methods, 
the fluid mesh moves and deforms to follow the structure. The Arbitrary Lagrangian-Eulerian (ALE) formulation is often used for the fluid equations. This approach is common for blood/artery wall interactions (see e.g. [16]) and blood/myocardium interactions. For the blood/heart valve interaction, some results have been reported using the moving domain method for both natural and prosthetic valves [5, 29, 37]. However, the large structural displacements of the valve require significant deformations of the fluid mesh and frequent re-meshing. Moreover, at the closure of the valve, the change of topology makes it difficult to properly define the ALE formulation. Our approach used a fixed domain method, which typically are more versatile at simulating FSI problems involving large structural displacements with possible topological changes. In this method, the fluid and structure meshes are independent.

The Fictitious Domain formulation (FD) is used in this work to couple the fluid and structure. The FD method, based on a variational approach, enforces the coupling conditions (7) by means of Lagrange multipliers located on the fluid-structure interface $\Sigma[2,11,41,40,14]$ or in the structure volume $\Omega^{\mathrm{s}}[48]$. For the structure model considered in this work, the two approaches are equivalent since $\Omega^{\mathrm{s}} \equiv \Sigma$. The coupled variational problem is discretized with the finite element method and solved with the partitioned scheme proposed in [14]. A strong Dirichlet-Neumann coupling has been realized by means of a fixed point algorithm between two independent solvers-one for the fluid and the other for the structure. The Aitken method is used to accelerate the convergence of the fixed point iterations [28].

A further improvement of the FSI model has been accomplished by adding detailed contact conditions in order to ensure impenetrability between the leaflets at the closure of the valve [30]. Details on the implementation of the contact algorithm in our fluid-structure procedure are reported

in [1]. This algorithm has been tested for a wide-range of physiological conditions, and demonstrates physiologic values for opening and closing times of the valve, total ejection time, and peak velocity of the jet. The results obtained from this approach are also comparable with the ones obtained, for example in $[10,31]$, with other numerical approaches.

\subsection{Computation of LCS}

The identification of LCS can be effectively achieved from the computation of finite-time Lyapunov exponent (FTLE) fields. The FTLE fields are computed by postprocessing the results obtained from the FSI simulation. In practice, FTLE fields are typically computed by integrating dense meshes of Lagrangian particles and LCS are extracted as codimension-one structures that maximize the FTLE 
measure (see e.g. $[23,35,26]$ ). Due to complex geometries and highly transient flow conditions, care must be taken in computing LCS in cardiovascular applications. Below, practical considerations relevant to the computation of FTLE from blood flow data obtained by the finite element method are discussed. In particular, the handling of large data sets and efficient integration on unstructured velocity meshes is addressed. The focus is on 3D data; minor modifications are needed to address 2D data.

\subsubsection{Kinematic model}

An essential step in the computation of the FTLE field is the computation of particle trajectories. In reality, blood is a composition of water containing various dissolved substances and suspended cells. However, it is reasonable to treat blood as a homogenous fluid over the length scales considered given the small size of the cells and nearly uniform density of the suspension. Furthermore, the time scales considered are sufficiently short (on the order of 1 second) that inter-cellular interactions and diffusion may typically be neglected. For example the diffusion coefficient of platelets is estimated to be on the order of $10^{-6}$ to $10^{-7} \mathrm{~cm}^{2} / \mathrm{s}$ [6], whereas $\boldsymbol{u}$ is typically on the order of $10^{1}$ to $10^{2} \mathrm{~cm} / \mathrm{s}$. Therefore, it is reasonable to assume that advection dominates transport and the equation governing particle trajectories is

$$
\mathbf{x}(t+T)=\mathbf{x}(t)+\int_{t}^{T} \boldsymbol{u}(\mathbf{x}(\tau), \tau) d \tau
$$

When defining the FTLE, it is convenient to rewrite the solution to Eq. (8) as a mapping $\phi(\mathbf{x}, t, T): \mathbf{x}(t) \mapsto \mathbf{x}(t+T)$, as details of the trajectory itself are inconsequential. A discrete approximation for the spatial variation of this flow map can be obtained by integrating a mesh of particles, from which the linearization of the flow map can be computed. The FTLE is then obtained as

$$
\sigma(\mathbf{x}, t, T)=\frac{1}{\|T\|} \ln \left\|\frac{d \phi(\mathbf{x}, t, T)}{d \mathbf{x}}\right\|
$$

where the induced $L_{2}$ norm is used. It is not difficult to show (see [35]) that

$$
\|\mathbf{y}(t+T)-\mathbf{x}(t+T)\| \approx \exp ^{\sigma(\mathbf{x}, t, T) T}\|\mathbf{y}(t)-\mathbf{x}(t)\|
$$

for small $\|\mathbf{y}(t)-\mathbf{x}(t)\|$. Thus, strongly hyperbolic trajectories will have a high FTLE values.

The utility of LCS computations is founded on the building computational and experimental evidence that complex fluid motion encountered in nature is often dictated by locations of strong hyperbolicity, which force the dynamics of surrounding fluid to quickly converge or diverge. 
The computation of attracting LCS (obtained from FTLE fields when $T<0$ ) and repelling LCS (obtained from FTLE fields with $T>0$ ) enables us to understand the extent of this influence throughout the domain of the fluid.

\subsubsection{Data management}

Velocity data is specified over a tetrahedral mesh at times $t_{k}$, for $k=1, \ldots, N_{t}$. Typically, the mesh size or temporal resolution is such that the velocity data is too large for the entire time history to be loaded into memory at once. Velocity is interpolated linearly in time, thus only data for two time points need be loaded concurrently. Using this strategy, the window of data loaded into memory is regularly shifted as the integration of particle trajectories proceeds.

The FTLE mesh is defined by a Cartesian grid with resolution $N_{x}, N_{y}$ and $N_{z}$. A new mesh of particles is regenerated for each time the FTLE field is computed. Suppose $t_{r}, r=1, \ldots, N_{r}$, denotes the times at which the FTLE field is to be determined. A new mesh of $N_{\mathbf{x}}=N_{x} \times N_{y} \times N_{z}$ particles is released $N_{r}$ times. The total number of particles to be tracked, $N_{\mathbf{x}} \times N_{r}$, can be quite large. Thus, for each new window of velocity data loaded into memory, each release is processed over that time window sequentially to avoid high memory usage; for example, starting from $t_{k=1} \leq t_{r=1}$, the general algorithm would be as follows:

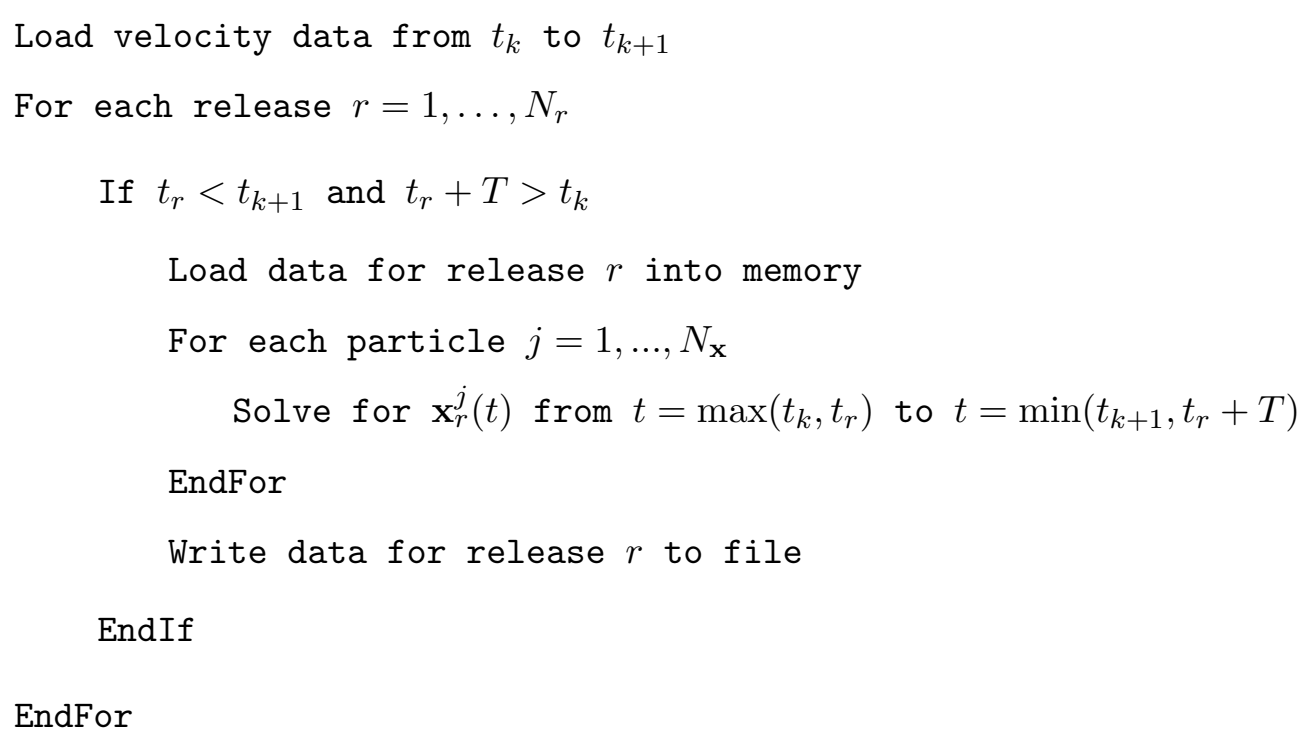

New velocity data is loaded as needed to integrate all FTLE releases. Here, we have assumed that $T>0$. Appropriate modifications should be made for backward-time integration, i.e. $T<0$, used to compute attracting LCS. 


\subsection{Parallelization}

Since the particles are non-interacting, the integration of particle trajectories can readily be parallelized. When $N_{r}$ is commensurate with the number of CPUs, $N_{p}$, the most straightforward parallelization is distributing each release to a separate process. This parallelization can be done in a cyclic fashion when $N_{r}>N_{p}$. When $N_{r} \ll N_{p}$, then it is more efficient to distribute blocks of particles to each process. For simulations where it is expected that particles will not readily exit the domain, a straightforward block partitioning of the FTLE mesh is reasonable. In applications where particles readily leave the domain, a cyclic domain decomposition or dynamic assignment of work is needed for load balancing.

\subsection{Velocity interpolation}

Let $\bar{e}(\mathbf{x}(t))$ define the element bounding the particle $\mathbf{x}(t)$ at time $t$. To interpolate the velocity at $\mathbf{x}(t)$, the velocities at the nodes of $\bar{e}(\mathbf{x}(t))$ are first interpolated in time by

$$
\boldsymbol{u}_{n}^{\bar{e}}(t)=\left[1-\delta_{t}\right] \boldsymbol{u}_{n}^{\bar{e}}\left(t_{k}\right)+\delta_{t} \boldsymbol{u}_{n}^{\bar{e}}\left(t_{k+1}\right)
$$

where $\boldsymbol{u}_{n}^{\bar{e}}$ denotes the velocity at node $n$ of element $\bar{e}, t \in\left[t_{k}, t_{k+1}\right]$ and $\delta_{t}=\left[t-t_{k}\right] /\left[t_{k+1}-t_{k}\right]$.

For spatial interpolation, natural coordinates are utilized, i.e. a coordinate transformation $\mathbf{x} \mapsto \boldsymbol{\xi}$ is employed so that the nodes of $\bar{e}(\mathbf{x}(t))$ are located at $(0,0,0),(1,0,0),(0,1,0),(0,0,1)$, see Fig. 3. In this frame, the velocity at location $\boldsymbol{\xi}=(\xi, \eta, \zeta)$ inside the element is given by

$$
\boldsymbol{u}(\boldsymbol{\xi})(t)=\boldsymbol{u}_{1}^{\bar{e}}(t)+\left[\boldsymbol{u}_{2}^{\bar{e}}(t)-\boldsymbol{u}_{1}^{\bar{e}}(t)\right] \xi+\left[\boldsymbol{u}_{3}^{\bar{e}}(t)-\boldsymbol{u}_{1}^{\bar{e}}(t)\right] \eta+\left[\boldsymbol{u}_{4}^{\bar{e}}(t)-\boldsymbol{u}_{1}^{\bar{e}}(t)\right] \zeta
$$

Note that $\boldsymbol{u}(\mathbf{x})(t)=\boldsymbol{u}(\boldsymbol{\xi})(t)$, so to evaluate $\boldsymbol{u}(\mathbf{x})(t)$ from Eq. (12), the mapping $\mathbf{x} \mapsto \boldsymbol{\xi}$ is needed.

For tetrahedral elements, the transformation $\boldsymbol{\xi} \mapsto \mathbf{x}$ takes the same form as Eq. (12), that is,

$$
\mathbf{x}(\boldsymbol{\xi})=\mathbf{x}_{1}^{\bar{e}}+\left[\mathbf{x}_{2}^{\bar{e}}-\mathbf{x}_{1}^{\bar{e}}\right] \xi+\left[\mathbf{x}_{3}^{\bar{e}}-\mathbf{x}_{1}^{\bar{e}}\right] \eta+\left[\mathbf{x}_{4}^{\bar{e}}-\mathbf{x}_{1}^{\bar{e}}\right] \zeta
$$

where $\mathbf{x}_{n}^{\bar{e}}$ denotes the coordinates of node $n$ of element $\bar{e}$. This mapping can be inverted to provide $\xi, \eta$, and $\zeta$ as a function of the nodal coordinates, $\mathbf{x}_{n}^{\bar{e}}$ (see [24]). These values can then be plugged into Eq. (12) to solve for $\boldsymbol{u}(\mathbf{x})(t)$.

Therefore, to interpolate the velocity at location $\mathbf{x}$ in physical coordinates we must (1) find the element containing $\mathbf{x}(t)$ at time $t$ and (2) transform $\mathbf{x}$ to the element's natural coordinates. Since the particle at $\mathbf{x}(t)$ is constantly moving, in practice we work from a "guess", $e_{g}$, for the element we believe contains $\mathbf{x}(t)$ at time $t$ and (1) transform $\mathbf{x}$ to the natural coordinate frame of $e_{g},(2)$ 
evaluate if indeed $\bar{e}(\mathbf{x}(t))=e_{g}$, and (3) interpolate velocity if $\bar{e}(\mathbf{x}(t))=e_{g}$, otherwise restart at step (1) using a new guess element, which is chosen as described next.

\subsection{Element search}

Another advantage of mapping $\mathbf{x}(t)$ to natural coordinates is to simplify the search for $\bar{e}(\mathbf{x}(t))$. In the natural coordinates of $e_{g}, \mathbf{x}$ is bounded by $e_{g}$ if all of the following are satisfied:

$$
\xi \geq 0, \quad \eta \geq 0, \quad \zeta \geq 0, \quad \text { and } \quad 1-\xi-\eta-\zeta \geq 0
$$

If any of these conditions are not satisfied, then a neighboring element is considered. Whichever condition is not satisfied indicates the neighbor to consider. For example, if $\xi<0$, then the neighbor sharing the $\xi=0$ face is used as the next guess. If more than one condition is not satisfied, the condition that is in greatest violation is used to determine the neighbor to search. In this way, the search progresses in the direction of the point and will readily converge to $\bar{e}(\mathbf{x}(t))$. Performing a local search in this manner requires adjacency information; this is usually available from the velocity mesh generation step, or can be computed as a preprocessing step. Also note that elements along the boundary of the velocity domain will have faces that are not shared with another element. If the local search tries to progress in the direction of a boundary face, the search returns a failure. When integrating a particle's trajectory, this occurs when the particle leaves the domain. When a particle leaves the domain, the integration for that particle is terminated.

\subsection{Initialization}

The efficiency of the local search method depends on the choice of the guess element. Before particles are integrated, the element containing each point in the FTLE mesh is determined. Roughly, this is performed as follows:

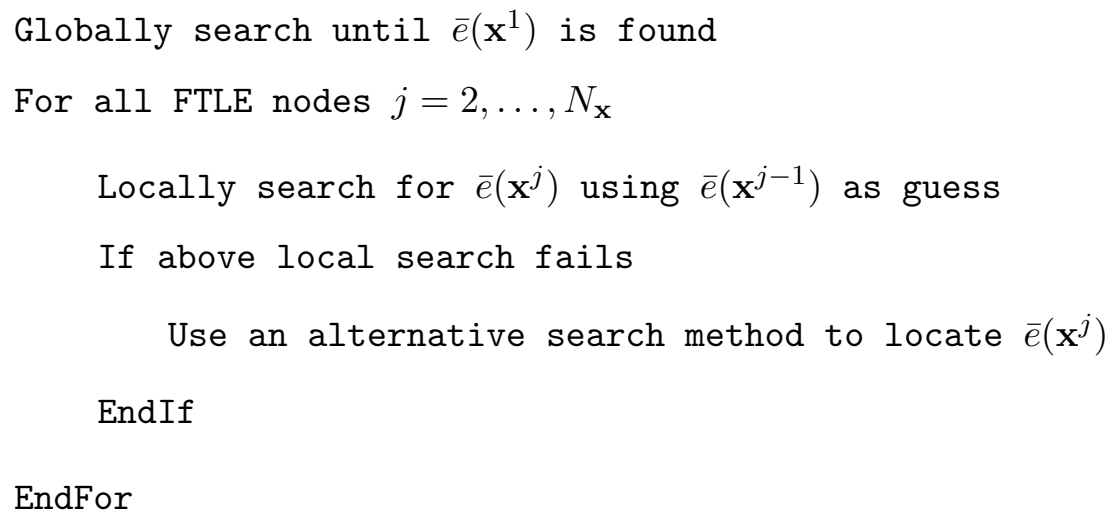


We have assumed that $\mathbf{x}^{j}$ neighbors $\mathbf{x}^{j-1}$ since the mesh is structured. If the intersection of the FTLE domain and velocity domain forms a non-convex set, then the local search protocol can fail for FTLE nodes located inside the velocity domain. Alternative search method(s) should be used when the local search fails (e.g. herein we used a local search from a seed location, but a global search, or other alternatives, may be needed for more complicated models). If any nodes in the FTLE mesh are located outside of the velocity domain, the alternative search method should be relatively robust at distinguishing these points while maintaining low computational expense. Additionally, one should ensure that the local search for $\bar{e}\left(\mathbf{x}^{j}\right)$ uses an appropriate guess element if $\mathbf{x}^{j-1}$ is located outside of the velocity domain.

\subsection{Integration}

The trajectory of each point in the FTLE mesh is obtained by solving Eq. (8). The integral on the right-hand side is evaluated using the 4th-order Runge-Kutta scheme or the adaptive timestepping of the Runge-Kutta-Fehlberg method. The nominal time step size $h$ (or limits for the adaptive stepping) is chosen from consideration of the CFL condition

$$
\frac{h \boldsymbol{u}_{\max }}{\bar{d}}<C
$$

where $\bar{d}$ is the nominal edge size of the tetrahedral elements in the velocity field mesh and $\boldsymbol{u}_{\max }$ is the maximum velocity occurring in space and time. Typically, $0.1 \leq C \leq 1$ is used in determining the nominal step size.

Once $\bar{e}\left(\mathbf{x}^{j}\right)$ has been computed for all FTLE nodes located inside the velocity domain (§2.6), the integration of particles from $\mathbf{x}^{j}$ can readily proceed since the element bounding each particle is known. As the integration proceeds, the variation in the interpolation location (particle position) from one step to the next is small enough that the local element search typically only proceeds over, at most, a few elements, making the element search highly efficient.

Due to the discrete nature of the integration, special techniques are often needed to ensure particles do not cross vessel walls or get stuck near a no-slip wall. These problems are often minimized if boundary layer meshing is used when generating the velocity field mesh. To prevent particles from crossing the vessel wall, inlet and outlet faces can be tagged. When the local element search fails for a particle, one can difference the position of the particle before and after the integration step to determine if it crossed a vessel wall or an inlet/outlet face. If it is determined that the particle crossed a vessel wall, the integration procedure or interpolation can be modified. 
For example, the step size can be adapted or the component of the interpolated velocity vector in the direction of the wall can be removed or modified (used herein). Furthermore, higher order basis functions can be used when interpolating inside wall (no-slip boundary) elements to prevent particles stagnating near the wall due to linear interpolation underestimating tangential flow.

\subsubsection{LCS extraction}

Commonly, LCS are identified by visual inspection from the FTLE field. However, it is convenient to parameterize these structures, especially when they are used for further postprocessing or analysis. In order to parameterize the LCS, we use the technique described in [33]. Based on the definitions presented in [35, 26], LCS are co-dimension one objects (lines in 2D, surfaces in 3D) that, generally speaking, satisfy the two conditions for each point $\mathrm{x}$ on the LCS:

D1 The gradient of $\sigma(\mathbf{x}, t, T)$ is aligned with the LCS.

D2 The principle direction (eigenvector) corresponding to the minimum eigenvalue of the Hessian of $\sigma(\mathbf{x}, t, T)$ is orthogonal to the LCS.

For these two conditions, the first and second derivatives of $\sigma(\mathbf{x}, t, T)$ must be computed from the FTLE field. When the field is "noisy" or contains many LCS, approximating these derivatives can be challenging and smoothing the field, or refining the computation of the FTLE field near the LCS, becomes necessary.

Let $\mathbf{e}_{\min }(\mathbf{x})$ denote the the direction of minimum principle curvature (D2) in order to define the function $\alpha(\mathbf{x})=\nabla_{\mathbf{x}} \sigma \cdot \mathbf{e}_{\min }$. LCS can be defined by the points $\mathbf{x}$ such that $\alpha(\mathbf{x})=0$. In practice, one may check for certain conditions at these points and exclude sections of the $\alpha(\mathbf{x})=0$ level set. For example, one can extract "well-defined" ridges by requiring the magnitude of the minimum principal curvature be above a certain threshold. For the case of $3 \mathrm{D}$ problems, one should also check that the finite-time deformation tensor

$$
\left(\frac{d \phi(\mathbf{x}(t), t, T)}{d \mathbf{x}(t)}\right)^{\mathrm{T}}\left(\frac{d \phi(\mathbf{x}(t), t, T)}{d \mathbf{x}(t)}\right)
$$

has only a single negative eigenvalue (see Def. 2.1 in [26]). 


\section{Results}

\subsection{Two-dimensional simulation}

Although flow through the aortic valve in inherently 3D, it is instructive to visualize the structure of the flow in the simpler, 2D case. For illustration purposes, flow through an idealized 2D heart valve model was investigated. The geometry of the aortic root shown in Fig. 4 was used. The fluid domain was discretized by 5902 triangular elements. The two leaflets are of length $1.728 \mathrm{~cm}$ and each one was discretized by 54 Hermit elements. We considered a rather stiff valve characterized by a thickness of $0.1 \mathrm{~cm}$ and a flexual stiffness $E I=3 \mathrm{gcm}^{3} / \mathrm{s}^{2}$. At the inflow boundary, the flow profile shown in Fig. 4 was imposed. At the outflow boundary, a resistance boundary condition was prescribed, with resistance $R=3500$ dyne $\cdot \mathrm{s} / \mathrm{cm}^{3}$. Flow was simulated over multiple cardiac cycles with a time-step size of $2 \times 10^{-3} \mathrm{~s}$ to reach a periodic regime.

The velocity field following peak systole is shown in Fig. 5(a). Figure 5(b) shows the backward time FTLE field following peak systole, which approximately corresponds to the point in time where the jet is near maximum strength and fully-developed. As blood is rapidly ejected from the valve, flow separation occurs near the tips of the leaflets. The repelling LCS extending from the leaflets is a material boundary between the jet of ejected blood and the region of separated, recirculating flow. Additional other LCS can be detected in the separated flow regions, which bound vortical structures (in the same sense as see [34]) of recirculating flow.

\subsection{Three-dimensional simulation}

For the 3D simulation we considered part of the idealized 3D geometry of the aortic root described in [38]. The fluid and structure computational domains, as well as the pulsatile periodic flow waveform imposed at the inlet, are shown in Fig 6 . To take into account the downstream vasculature, a resistance $R=500$ dyne $\cdot \mathrm{s} / \mathrm{cm}^{5}$ was prescribed as the outlet boundary condition. Fluid and structure domains were discretized, respectively, by 216692 tetrahedral and 4814 quadrilateral elements. Leaflets were characterized by a density $\rho^{\mathrm{s}}=1.2 \mathrm{~g} / \mathrm{cm}^{3}$, an elastic modulus $E=10^{6}$ dyne $/ \mathrm{cm}^{2}$, a Poisson ratio of 0.3 and a thickness of $0.05 \mathrm{~cm}$.

Flow was simulated over 2 cardiac cycles with a time-step size of $2.5 \times 10^{-4} \mathrm{~s}$ and data from the last cardiac cycle was used for analysis. The velocity field during systole is shown in Fig. 7 . For visualization purposes, only vectors from a subset of the nodes of the finite-element mesh are plotted. Also shown is the location of the valve over time. 
The backward-time FTLE field was computed in the vicinity of the 3D aortic valve from the FSI simulation data shown in Fig. 7. A section of the FTLE field, spanning from the root of the valve to $1 \mathrm{~cm}$ downstream from the leaflet tips when fully opened, is shown in Fig. 8 at four time instances during systole. This cross-section roughly corresponds to the location where minimal cross-sectional area of the jet occurs shortly after peak systole; however this location of minimum area continually changes. Initially, a single attracting LCS, shown as the white surface in panels (a) and (b) of Fig. 8, bounds the region of blood being ejected as the valve opens. Near peak systole, flow separation from the leaflets occurs. The LCS shown in panels (c) and (d) of Fig. 8 capture these separation profiles. Note that attracting LCS approximately coincide with each leaflet and thus these LCS appear fragmented along the leaflet when visualizing both structures together. From the LCS, we can see that as the flow separates from the leaflets, it rolls up along the edges. A similar separation profile was identified in studying flow separation in the carotid bifurcation in [36].

Since the LCS extending from the leaflets defines the boundary of the jet, we can readily obtain an accurate measure of jet area from the LCS. This was done at a location $1 \mathrm{~cm}$ distal to the leaflet tips in the fully open state. The location chosen roughly corresponds to the region where the minimal cross-sectional area of the jet occur. Even if a more accurate selection could be done, for example through an optimization algorithm, we consider this level of accuracy reasonable in view of the uncertainties of the model. The cross-section of the FTLE field at this location is shown in Fig. 9(a) during systole. We used the algorithm described in $§ 2.7 .1$ to extract a parametrization for the cross-section of LCS at a series of points in time. The area bound by these curves was then computed at these time points and the results are shown in Fig. 9(b). Time 0 corresponds to the start of systole (the opening of the valve) and peak systole occurs after approximately 0.11 seconds. The size of the jet at this cross-section reaches approximately $1.63 \mathrm{~cm}^{2}$, with the peak cross-sectional area occurring around $t=0.17 \mathrm{~s}$, which does not correspond to peak systole. The reason for this is twofold. First, it takes a finite amount of time for the jet to develop. Secondly, it takes a finite amount of time for the jet to propagate downstream.

It is interesting to compare the (precise) size of the jet obtained from LCS with that obtained using the continuity equation, a popular formula typically used in clinical practice to estimate the EOA. The continuity equation states that the flow rate in the left ventricular outflow tract (LVOT) equals the one in the vena contracta (VC), that is

$$
\mathrm{EOA} \times \mathrm{VTI}_{\mathrm{VC}}=\mathrm{A}_{\mathrm{LVOT}} \times \mathrm{VTI}_{\mathrm{LVOT}}
$$


The quantity $\mathrm{VTI}_{\mathrm{VC}}$ is the velocity time integral at the level of the vena contracta, while $\mathrm{A}_{\mathrm{LVOT}}$ and $\mathrm{VTI}_{\text {LVOT }}$ represent, respectively, the subvalvular cross-sectional area (see Fig. 1) and the corresponding velocity time integral. In clinical practice, the VTI on a prescribed cross-section is obtained from an integration in time of Doppler velocity measurements over the systolic phase of the cardiac cycle. Therefore, to estimate the EOA the following integral relationship is evaluated

$$
\mathrm{EOA}=\frac{\mathrm{A}_{\mathrm{LVOT}} \mathrm{VTI}_{\mathrm{LVOT}}}{\mathrm{VTI}_{\mathrm{VC}}}
$$

where we can think of the numerator as the stoke volume and the denominator as the integral of the velocity at the center of the vena contracta of the jet. Evaluating equation (16) from the 3D FSI velocity data at the cross-sectional location in Fig. 9 gives EOA $=1.53 \mathrm{~cm}^{2}$. This value is in the range of the measurements obtained with LCS (see Fig. 9(b)) but underestimates the true (peak) area of about $5 \%$.

\section{Discussion}

In this paper we demonstrated a novel approach for understanding the geometry and dynamics of the jet produced by the aortic valve. In particular, flow through the aortic valve was simulated using a robust FSI scheme to provide highly resolved velocity data in the vicinity of the aortic valve over serval cardiac cycles. This data was used to perform LCS computations, which revealed flow separation from the valve leaflets during systole, and correspondingly, the boundary between the jet of ejected fluid and the regions of separated, recirculating flow.

Advantages of computing LCS in multi-dimensional FSI models of the aortic valve are twofold. For one, the quality and effectiveness of existing clinical indices used to measure aortic jet size can be tested in different stenotic scenarios by taking advantage of the accurate measure of the jet area derived from LCS. Secondly, as an ultimate goal, a reliable computational framework for the assessment of the aortic valve stenosis could be developed. For this latter point, however, it must be recognized that a precise knowledge of the mechanical and geometrical properties of the system could be needed for patient-specific medical planning.

As shown in $\S 3$, LCS provide clear, unambiguous boundaries to the jet. Currently, in practice, the size of the aortic jet is measured by the EOA. However, the computation of the EOA provides little insight into the actual geometry or dynamics of the jet since these computations are based on simplifying assumptions. It is conceivable, and most likely, that jets with dissimilar geometry and dynamics could produce similar indices. Since the local blood flow mechanics (recirculation, 
separations, mixing, etc.) is known to strongly influence pathophysiology (see e.g. [25, 45]), the computation of LCS has the clear benefit of providing insight into these conditions. Furthermore, as shown in $\S 3$, clinical indices, such as the one based on the continuity equation, provide only averaged information.

As for practical concerns, note that to obtain the jet area, the entire 3D FTLE field is not needed. Only the section of the FTLE field at the location where the area measurement is to be made is needed. This is an important consideration since it greatly reduces the computational cost needed to compute the jet area. Additionally, as shown above, automatic extraction of the LCS is possible, which, for one, removes user bias (which is currently an issue for EOA calculations), and secondly, makes these methods potentially accessible to the medical community either from numerical or clinical data. Additionally, a better understanding the 3D flow geometry of the jet could enable improved techniques for measuring flow conditions or developing more effective metrics for accessing the severity of AS from current and subsequent modalities and technologies.

\section{Aknowledgements}

The research leading to these results has received funding from the European Community's Seventh Framework Programme (FP7/2007-2013) under grant agreement n 224495 (euHeart project) and from the associated team "Cardio" INRIA-Stanford. The authors would like to sincerely thank Irene Vignon-Clementel for fruitful discussions and her help promoting this collaboration.

\section{References}

[1] M. Astorino, J.-F. Gerbeau, Pantz O., and K.-F. Traoré. Fluid-structure interaction and multibody contact: Application to the aortic valves. Computer Methods in Applied Mechanics and Engineering, 198(45-46):3603-3612, 2009.

[2] F. Baaijens. A fictitious domain/mortar element method for fluid-structure interaction. International Journal for Numerical Methods in Fluids, 35:743-761, 2001.

[3] H. Baumgartner. Hemodynamic assessment of aortic stenosis. Journal of the American College of Cardiology, 47(1):138-140, 2006.

[4] H. Baumgartner, J. Hung, J. Bermejo, J.B. Chambers, A. Evangelista, B.P. Griffin, B. Iung, C.M. Otto, P.A. Pellikka, and M. Quinones. Echocardiographic assessment of valve steno- 
sis: EAE/ASE recommendations for clinical practice. Journal of The American Society of Echocardiography, 22(1):1-23, 2009.

[5] C.J. Carmody, G. Burriesci, I.C. Howard, and E.A. Patterson. An approach to the simulation of fluid-structure interaction in the aortic valve. Journal of Biomechanics, 39:158-169, 2006.

[6] C. G. Caro, T. J. Pedley, R. C. Schroter, and W. A. Seed. The Mechanics of the Circulation. Oxford University Press, Oxford, 1978.

[7] P. Causin, N. Diniz dos Santos, J.F. Gerbeau, C. Guiver, and P. Metier. An embedded surface method for valve simulation: Application to stenotic aortic valve estimation. ESAIM: Proceedings, 14:48-62, 2005.

[8] J.B. Chambers, D.C. Sprigings, T. Cochrane, J. Allen, R. Morris, M.M. Black, and G. Jackson. Continuity equation and Gorlin formula compared with directly observed orifice area in native and prosthetic aortic valves. British Heart Journal, 67:193-199, 1992.

[9] D. Chapelle and K.J. Bathe. The Finite Element Analysis of Shells - Fundamentals. Springer Verlag, 2003.

[10] J. de Hart. Fluid-Structure Interaction in the Aortic Heart Valve: a three-dimensional computational analysis. PhD thesis, Technische Universiteit Eindhoven, 2002.

[11] J. de Hart, F. Baaijens, G.W.M. Peters, and P.J.G. Schreurs. A computational fluid-structure interaction analysis of a fiber-reinforced stentless aortic valve. Journal of Biomechanics, 36:699-712, 2003.

[12] J. de Hart, G.W.M. Peters, P.J.G. Schreurs, and F.P.T. Baaijens. A three-dimensional computational analysis of fluid-structure interaction in the aortic valve. Journal of Biomechanics, $36: 103-112,2003$.

[13] C.G. DeGroff, R. Shandas, and L. Valdes-Cruz. Analysis of the effect of flow rate on the doppler continuity equation for stenotic orifice area calculations: A numerical study. Circulation, 97:1597-1605, 1998.

[14] N. Diniz dos Santos, J.-F. Gerbeau, , and J.-F. Bourgat. A partitioned fluid-structure algorithm for elastic thin valves with contact. Computer Methods in Applied Mechanics and Engineering, 197:1750-1761, 2008. 
[15] J.G. Dumesnil and A.P. Yoganathan. Theoretical and practical differences between the Gorlin formula and the continuity equation for calculating aortic and mitral valve areas. The American Journal of Cardiology, 67:1268-1272, 1991.

[16] M.A. Fernández and J.-F. Gerbeau. Fluid structure interaction problems in haemodynamics, chapter 9. Springer Verlag, 2009. in Cardiovascular Mathematics, L. Formaggia, A. Quarteroni, A. Veneziani editors.

[17] D. Garcia, P. Barenbrug, P. Pibarot, A. Dekker, F.H. van der Veen, J.G. Maessen, J.G. Dumesnil, and L.G. Durand. A ventricular-vascular coupling model in presence of aortic stenosis. American Journal of Physiology, 288:H1874-H1884, 2005.

[18] D. Garcia and L.G. Durand. Wiley Encyclopedia of Biomedical Engineering, chapter Aortic stenosis and systemic hypertension, Modeling of. John Wiley \& Sons, Inc., 2006.

[19] D. Garcia and L. Kadem. What do you mean by aortic valve area: Geometric orifice area, effective orifice area or Gorlin area? Journal of Heart Valve Disease, 15:601-608, 2006.

[20] D. Garcia, P. Pibarot, J.G. Dumesnil, F. Sakr, and L.G. Durand. Assessment of aortic valve stenosis severity: A new index based on the energy loss concept. Circulation, 101:765-771, 2000.

[21] R. Gorlin and S.C. Gorlin. Hydraulic formula for calculation of the area of the stenotic mitral valve, other cardiac valves, and central circulatory shunts. American Heart Journal, 41:1-29, 1951.

[22] C. Guivier, V. Deplano, and P. Pibarot. New insights into the assessment of the prosthetic valve performance in the presence of subaortic stenosis through a fluid-structure interaction model. Journal of Biomechanics, 40:2283-2290, 2007.

[23] G. Haller. Distinguished material surfaces and coherent structures in three-dimensional fluid flows. Physica D, 149(4):248-277, 2001.

[24] D.N. Kenwright and D.A. Lane. Interactive time-dependent particle tracing using tetrahedral decomposition. IEEE Transactions on Visualization and Computer Graphics, 2(2):120-129, 1996. 
[25] D.N. Ku, D.P. Giddens, C.K. Zarins, and S. Glagov. Pulsatile flow and atherosclerosis in the human carotid bifurcation: positive correlation between plaque location and low and oscillating shear-stress. Arteriosclerosis, 5:293-302, 1985.

[26] F. Lekien, S. C. Shadden, and J. E. Marsden. Lagrangian coherent structures in $n$-dimensional systems. Journal of Mathematical Physics, 48(6):065404, 2007.

[27] D.M. McQueen and C.S. Peskin. Heart simulation by an immersed boundary method with formal second-order accuracy and reduced numerical viscosity. In H. Aref and J.W. Phillips, editors, Mechanics for a New Mellennium, 2001.

[28] D. P. Mok, W. A. Wall, and E. Ramm. Accelerated iterative substructuring schemes for instationary fluid-structure interaction. In K.J. Bathe, editor, Computational Fluid and Solid Mechanics, pages 1325-1328. Elsevier, 2001.

[29] J.-D. Müller. Heart valve fluid-structure interaction using weak coupling. In P Nithiarasu, R Löhner, and R. van Loon, editors, CMBE09. Wiley, 2009.

[30] O. Pantz. A frictionless contact algorithm for deformable bodies. Preprint Ecole Politecnique, 2007.

[31] A. Ranga, O. Bouchot, R. Mongrain, P. Ugolini, and C. Raymond. Computational simulations of the aortic valve validated by imaging data: evaluation of valve-sparing techniques. Interactive Cardio Vascular and Thoracic Surgery, 5:373-378, 2006.

[32] K.L. Richards, S.R. Cannon, J.F. Miller, and M.H. Creawford. Calculation of aortic valve area by doppler echocardiography: A direct application of the continuity equation. Circulation, 73:964-969, 1986.

[33] S. C. Shadden. A dynamical systems approach to unsteady systems. PhD thesis, California Institute of Technology, 2006.

[34] S.C Shadden, J.O Dabiri, and J.E. Marsden. Lagrangian analysis of fluid transport in empirical vortex ring flows. Physics of Fluids, 18(4):047105, 2006.

[35] S.C. Shadden, F. Lekien, and J.E. Marsden. Definition and properties of Lagrangian coherent structures from finite-time Lyapunov exponents in two-dimensional aperiodic flows. Physica $D, 212(3-4): 271-304,2005$. 
[36] S.C. Shadden and C.A. Taylor. Characterization of coherent structures in the cardiovascular system. Annals of Biomedical Engineering, 36(7):1152-1162, 2008.

[37] F. Sotiropoulos and I. Borazjani. A review of the state-of -the-art numerical methods for simulating flow through mechanical heart valves. Medical and Biological Engineering and Computing, 47:245-256, 2009.

[38] T.-D. Tran. Construction d'un modéle $3 \mathrm{~d}$ comprenant les sinus de valsalva, la valve aortique et l'arche aortique. Master's thesis, École Polytechnique Montreal, 2006.

[39] R. van Loon. Towards computational modelling of aortic stenosis. Communications in Numerical Methods in Engineering, 2009.

[40] R. van Loon, P. D. Anderson, and F. N. van de Vosse. A fluid-structure interaction method with solid-rigid contact for heart valve dynamics. Journal of Computational Physics, 217(2):806$823,2006$.

[41] R. van Loon, P.D. Anderson, J. De Hart, and F.P.T. Baaijens. A combined fictitious domain/adaptative meshing method for fluid-structure interaction in heart valves. International Journal for Numerical Methods in Fluids, 46:533-544, 2004.

[42] I. E. Vignon-Clementel, C. A. Figueroa, K. E. Jansen, and C. A. Taylor. Outflow boundary conditions for three-dimensional finite element modeling of blood flow and pressure in arteries. Computer Methods in Applied Mechanics and Engineering, 195(29-32):3776-3796, 2006.

[43] E.J. Weinberg and M.R. Kaazempur-Mofrad. On the constitutive models for heart valve leaflet mechanics. Cardiovascular Engineering, 5(1):37-43, 2005.

[44] E.J. Weinberg and M.R. Kaazempur-Mofrad. Transient, three-dimensional, multiscale simulations of the human aortic valve. Cardiovascular Engineering, 7(4):140-155, 2007.

[45] D.M. Wootton and D.N. Ku. Fluid mechanics of vascular systems, diseases, and thrombosis. Annual Review of Biomedical Engineering 1:299-329, 1999.

[46] Z. Xu, N. Chen, S.C. Shadden, J.E. Marsden, M.M. Kamocka, E.D. Rosen, and M. Alber. Study of blood flow impact on growth of thrombi using a multiscale model. Soft Matter, 5:769-779, 2009. 
[47] F. Yilmaz and M.Y. Gundogdu. A critical review on blood flow in large arteries: Relevance to blood rheology, viscosity models, and physiologic conditions. Khorea-Ausrtalia Rheology Journal, 20(4):197-211, 2008.

[48] Z. Yu. A DLM/FD method for fluid/flexible-body interactions. Journal of Computational Physics, 207(1):1-27, 2005. 
List of Figures 


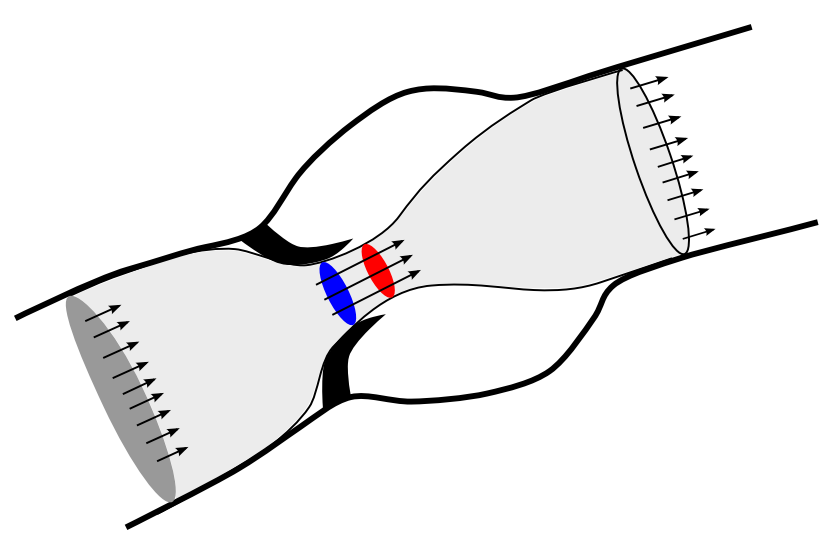

Figure 1: Schematic representation of the aortic valve and of the corresponding jet flow (light grey). In dark grey the subvalvular cross-sectional area, in blue the geometric orifice area (GOA), in red the effective orifice area (EOA).

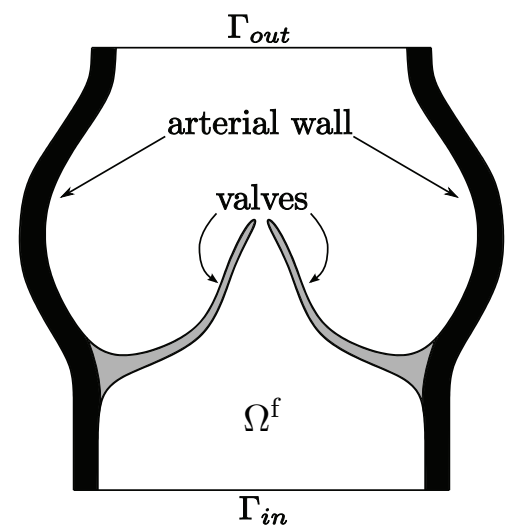

Figure 2: Schematic representation of the fluid-structure model. 

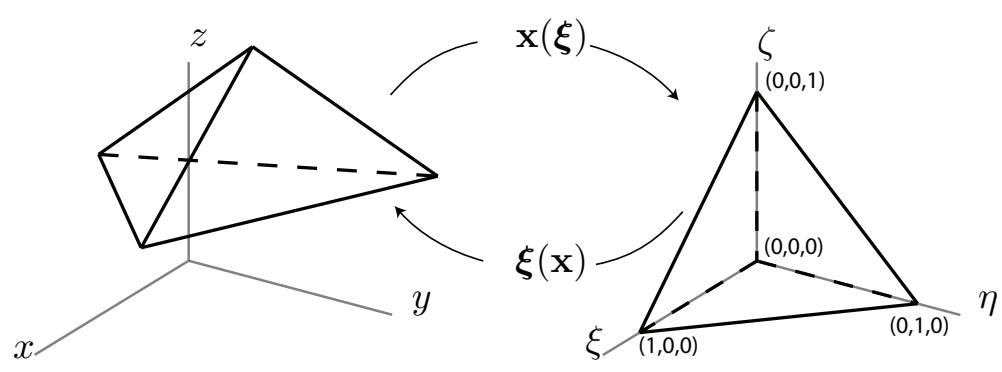

Figure 3: Transformation of tetrahedtral between physical coordinates $(x, y, z)$ and natural coordinates $(\xi, \eta, \zeta)$.
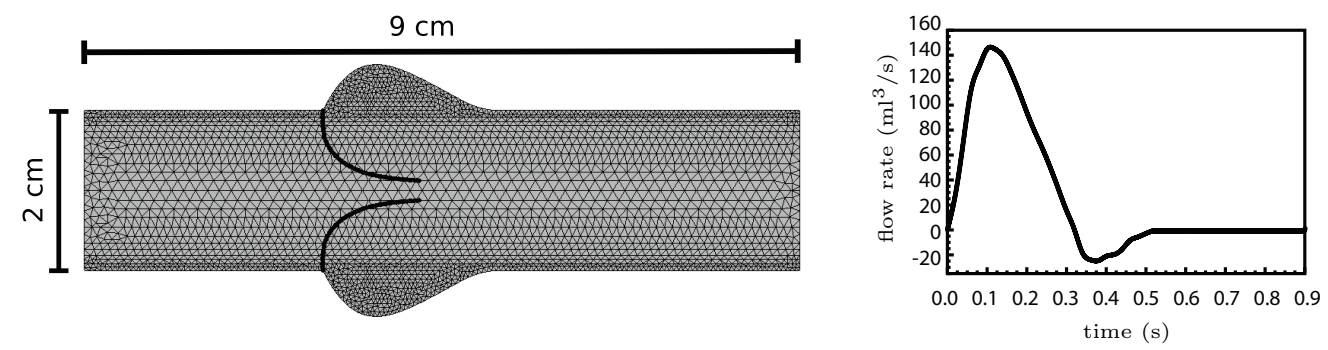

Figure 4: 2D computational domain and inflow waveform.

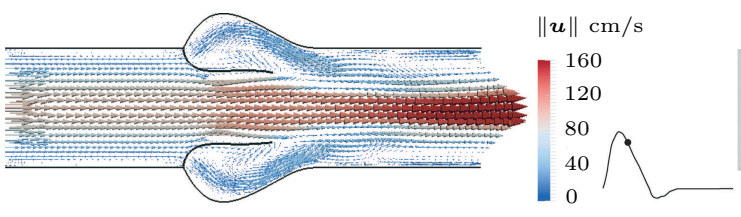

(a) Velocity field

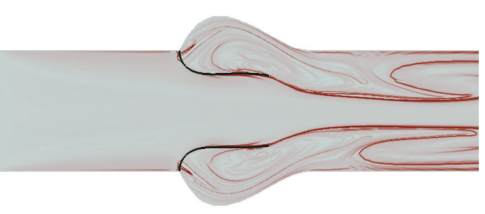

(b) FTLE field

Figure 5: (a) Velocity field from 2D simulation following peak systole $(t=0.16 \mathrm{~s}$ ) (b) FTLE field from 2D simulation following peak systole $(t=0.16 \mathrm{~s})$. Curves of high FTLE (dark) extending from leaflet tips demarcate boundary between jet and separated flow regions. 

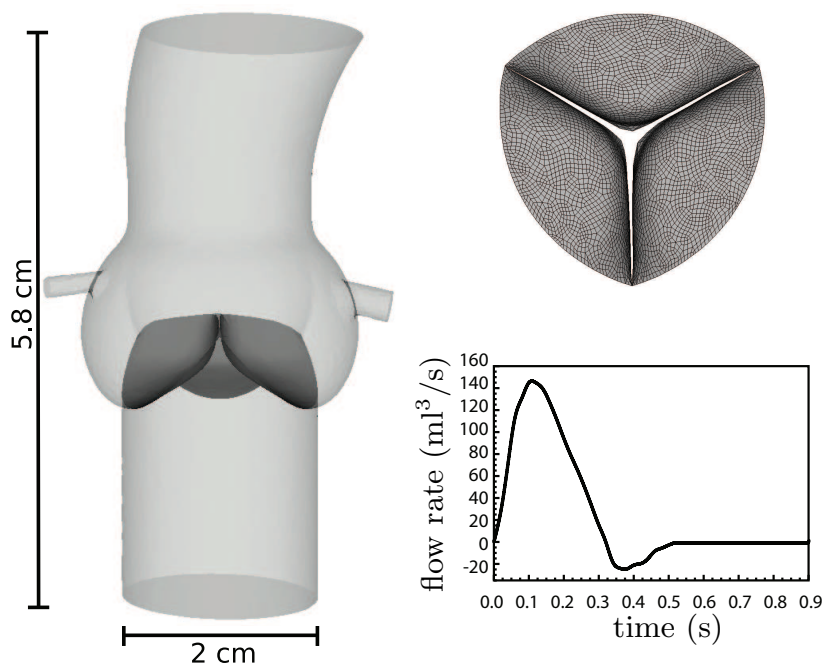

Figure 6: 3D computational domain and inflow waveform. Vessel are partially transparent and leaflets are opaque. A top view of the tricuspid valve is shown in upper right.

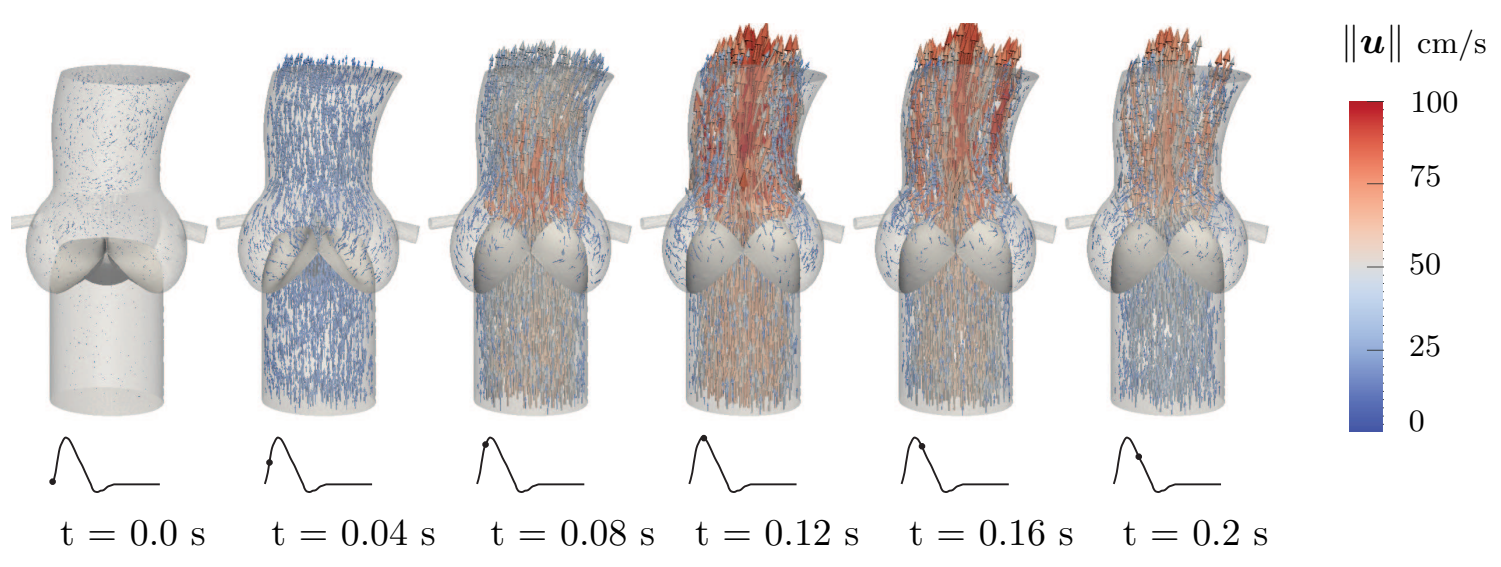

Figure 7: Velocity field during systolic phase from 3D simulation. 

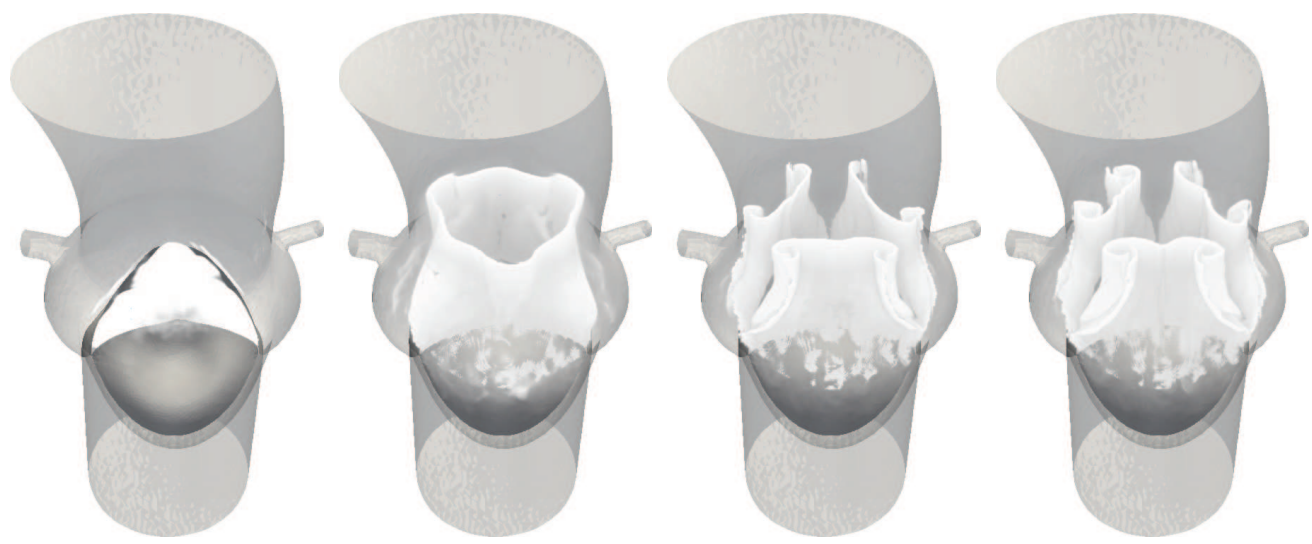

$t=0.05 \mathrm{~s}$

$t=0.08 \mathrm{~s}$

$t=0.11 \mathrm{~s}$

$t=0.14 \mathrm{~s}$

Figure 8: Backward time FTLE reveals attracting LCS (white) that bound the blood ejected from the tricuspid valve as it opens.

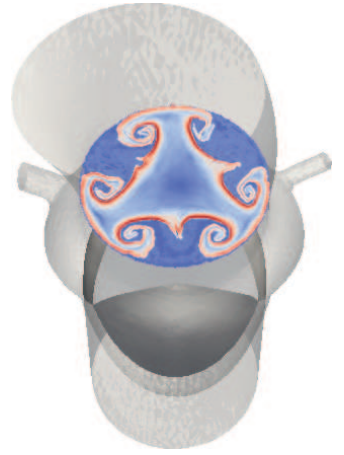

(a)

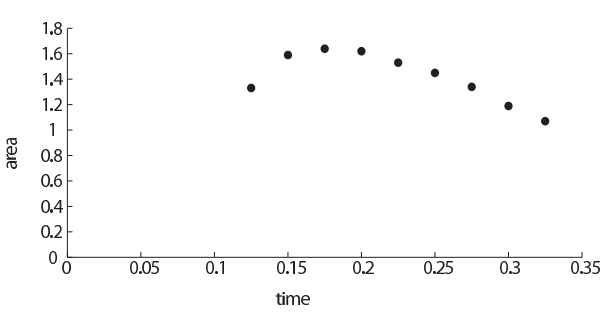

(b)

Figure 9: (a) Cross-section of 3D FTLE field at location $1 \mathrm{~cm}$ distal from aortic valve leaflet tips. (b) Jet area at location $1 \mathrm{~cm}$ distal from aortic valve leaflet tips over time. 